\title{
Recognition memory of spatial location information: Another failure to support automaticity
}

\author{
MOSHE NAVEH-BENJAMIN \\ Ben-Gurion University of the Negev, Beer-Sheva, Israel
}

\begin{abstract}
Hasher and Zacks (1979) claimed that spatial location information is automatically encoded. Evaluation of the empirical basis for this claim, however, casts doubt on some of the evidence for the automaticity position. This evaluation led to four experiments in which five criteria for testing the automaticity of cognitive processes were examined using a recognition task. Results of these experiments clearly show that recognition memory for spatial location information is influenced by intention, age of subjects, competing task loads, practice, and individual differences. The reported results, which extend those reported by Naveh-Benjamin (1987) for spatial location recall memory, are at odds with the claim that memory for spatial location information is exclusively mediated by automatic encoding processes. The concept of automaticity and the appropriateness of the criteria suggested for testing the automaticity of cognitive processes are discussed in light of the current results and recent findings on other features of the environment (e.g., frequency of occurrence) previously claimed to be automatically encoded.
\end{abstract}

Many studies in recent years have been conducted to identify automatic processes in cognition and to specify the details of their operation. Hasher and Zacks (1979) and their colleagues have argued that some basic operating characteristics of the information processing system support the inevitable memory encoding of some fundamental aspects of experience. Among them are frequency of occurrence, spatial location, and temporal order information. According to these researchers, these aspects continually register in memory at an optimal level.

Hasher and Zacks (1979) suggested six criteria that must be jointly satisfied to establish that a process, aspect, or attribute of experience is automatically encoded. According to these criteria, encoding of frequency, temporal order, and spatial location information should not be affected by intention, age, and simultaneous processing demands, or by practice and individual differences. The aspect of the environment most studied for its automaticity is the frequency with which events occur. Hasher and Zacks, among others, have amassed evidence about all the above-mentioned criteria for frequency coding. As a result, until recently, the claim for the automaticity of encoding frequency of occurrence seemed to have solid support (Hasher \& Zacks, 1984).

Two other aspects of the environment-spatial location and temporal order information-were thought to be en-

I would like to thank Eynat Cohen and Orly Hochwald for their help in data collection and analysis. I would also like to thank Robert R. Greene, Mary Joscelyn, Denise C. Park, and an anonymous reviewer for their comments on an earlier draft of the manuscript. Requests for reprints should be addressed to Moshe Naveh-Benjamin, Department of Behavioral Sciences, Ben-Gurion University of the Negev, P.O. Box 653, Beer-Sheva 84120, Israel. coded automatically, although they were not as thoroughly investigated as frequency of occurrence. Although Hasher and Zacks (1979) relied on a number of studies to support their automaticity claim for spatial location information, which is the focus of this paper, a careful look reveals that the results of some tests of criteria for automaticity are rather ambiguous, whereas other criteria have not been studied at all. In addition, results of a recent study (Naveh-Benjamin, 1987) were clearly not in line with the automaticity position. In the following section, some of the evidence about tests of the various criteria for automaticity of the encoding of spatial location information is reviewed.

\section{Effects of Intention}

A typical study cited by Hasher and Zacks (1979) for the criterion of no effect of intention is that by von Wright, Gebhard, and Karttunen (1975). These investigators presented subjects with a series of four-picture arrays and asked them to recall the pictures and the quadrant in which they were presented. Prewarning about the spatial location test did not affect performance. This study, however, is characterized by the problem that the incidental learning instructions used, in which subjects were told to expect a test on item information, were not the appropriate ones.

It has been argued recently in the context of frequency judgments (Fisk \& Schneider, 1984; Greene, 1984; Naveh-Benjamin \& Jonides, 1985, 1986) that giving instructions to pay attention and to expect a test on item information is inappropriate in establishing incidental learning of the memory attribute (frequency of occurrence in the above cases and spatial location information in the present case). With such instructions, subjects still pay 
attention and try to remember the information, so they might be using various strategies, including some in which spatial location of the objects is used to remember the items. As a consequence, such instructions cannot lead to a condition that is truly incidental. Therefore, comparing an incidental condition with an intentional condition (in which subjects are told that memory for both the items and their spatial location will be tested later) would not necessarily provide information about the effect of intention on memorizing the attribute. Instead, it was argued that, in addition to an intentional learning condition, a true incidental learning condition should be used in which subjects do not expect any memory test on the information presented, so that their learning could be considered truly incidental.

Some studies testing the effect of intention have shown either no effect (e.g., Zechmeister, McKillip, Pasko, \& Bespalec, 1975; McCormack, 1982) or an advantage of incidental over intentional learning (Schulman, 1973). All these studies, however, suffered from the use of inappropriate incidental learning conditions, as mentioned earlier.

Two studies, one by Mandler, Seegmiller, and Day (1977) and the other by Herman, Kolker, and Shaw (1982), actually claimed no effect of intention when using a true incidental learning condition. The results of these studies, however, present several problems. Although Mandler et al., claimed only "small loss" in memory for spatial location information under true incidental instructions, the results of both their experiments indicated that the performance of subjects run under such instructions was noticeably below that of subjects run under intentional instructions. Moreover, in their second experiment, subjects run under true incidental instructions performed significantly lower on the spatial memory task, in comparison with all other subjects.

The study by Herman et al. (1982) allowed the subjects a long time to study the stimuli, which might have induced the use of learning strategies even for the incidental learners. In addition, there was no lag between learning and testing. This might have prevented subjects in the intentional condition from employing various organizational and rehearsal strategies. Other studies actually have reported an advantage of intentional over incidental learning instructions (e.g., Acredolo, Pick, \& Olsen, 1975; Park \& James, 1983; Park \& Mason, 1982; Park, Puglisi, \& Lutz, 1982). All of these studies, however (except the one by Acredolo et al.), involved tasks that produce considerable interference because the same locations are $\alpha$ cupied by different items on successive presentations (see Light \& Zelinski, 1983).

\section{Effects of Age}

No definite evidence for the automaticity position emerges from the studies that investigated the effect of age on memory for spatial location information. Although at least one study (Mandler et al., 1977) showed no clear differences between children and adults (despite some differences between the groups of children as a function of their ages), other studies have shown developmental patterns in children (Park \& James, 1983) and changes between childhood and adulthood (von Wright et al., 1975). Still others have found that adults performed better than elderly persons in spatial location memory tasks (e.g., Light \& Zelinski, 1983; Park et al., 1982; Perlmutter, Metzger, Nezworski, \& Miller, 1981; Pezdek, 1983; Waddell \& Rogoff, 1981). It should be noted that neither type of material used nor number of spatial locations tested can account for these discrepant results. With one exception (Park et al., 1982), no study showed an effect of the interaction between age and learning instruction. The lack of an interaction effect may be looked at as supporting the claim for a lack of qualitative differences in the use of effective strategies by the various age groups.

\section{Effects of Simultaneous Processing Demands}

Most of the evidence bearing on automaticity comes from studies of the effects of competing task demands. Both theoretically and empirically, various investigators have argued that insensitivity to competing task demands is the hallmark of automaticity (Jonides, 1981; NavehBenjamin \& Jonides, 1984, 1986; Posner \& Snyder, 1975; Schneider \& Shiffrin, 1977; Shiffrin \& Schneider, 1977). The claim is that if an automatic process is assumed to require minimal mental capacity for its operation, simultaneous performance of a task with the automatic process should not affect the operation of the process.

A number of studies have used a dual task in the context of the encoding of spatial location information; the results of these experiments are mixed. For example, Park and Mason (1982) compared the performance of subjects who were instructed to memorize both item and location information with the performance of subjects who were asked to remember item information only. The results showed no decline in memory for item information by subjects who were asked to remember both item and spatial location information. These results appear to support the claim that attending to spatial location information does not require capacity, since there was no trading of item information for attribute information.

Park and James (1983) claimed that one reason for not finding such a trade-off was due to the relatively low task demands in Park and Mason's (1982) study. In supporting this position, they cited Park et al. (1982), who showed that using aged subjects, for whom task demands are apparently more difficult, resulted in a trade-off between item and spatial location information (see also Schulman, 1973). However, other studies that used a similar paradigm with children, for whom task demands should also be significant, have not shown a decline in item information under instructions to attend to both item and spatial location information (Mandler et al., 1977; Park \& James, 1983).

The results of all these studies are troublesome for two main reasons: First, it very possibly may be that item in- 
formation is mediated by accessing information about the spatial location of the item (Lovelace \& Southall, 1983), so one should not particularly expect that preparation for a spatial location test of the items themselves would impair item information performance. Second, there is no direct way of scaling the amount of cognitive demand induced by the different kinds of test preparation.

In summary, beyond showing mixed results, studies concerned with the effect of simultaneous processing on the encoding of spatial location information have not optimally tested the effect of competing demands.

\section{Effects of Practice}

Training for processing automatically coded information is not expected to improve performance. There have been no studies to date, however (except the one by Naveh-Benjamin, 1987, to be discussed later), that have tested explicitly the effect of practice on memory for spatial location information.

\section{Effects of Individual Differences}

Differences among people, such as in motivation, intelligence, prior relevant knowledge, and educational attainment, should not affect the automatic coding of information of spatial location. As in the case of the criterion of practice, no reported studies have directly tested this criterion in the context of automaticity of spatial location coding.

The above review makes it quite clear that the support for automatic encoding of spatial location information is far from solid. Some studies that seem to support the automaticity position have not used appropriate methodologies in testing the various criteria. For other criteria (e.g., effects of age), the results of the various studies conflict. In addition, some criteria have not been tested at all.

A recent study by Naveh-Benjamin (1987), in which the methodological problems mentioned above were corrected, actually tested all the criteria for automaticity of encoding of spatial location information. Naveh-Benjamin employed a task similar to the one used by Mandler et al. (1977), in which subjects were shown a matrix of 36 locations, 20 of which contained to-be-remembered items (small objects). In Experiment 1 , recall memory for spatial location information was significantly better under intentional than under pure incidental learning instructions. In addition, it was better in the adult group than in the elderly one. In Experiment 2 of Naveh-Benjamin's study, subjects who were involved in a more difficult concurrent task while studying the spatial location information had significantly poorer recall memory for location than subjects who studied it under an easier concurrent task. Results of another experiment showed recall memory for spatial location to be significantly affected by the amount of practice in the task. Finally, individual differences were shown to play a significant role in recall memory for spatial location.
The above results were obtained for measures of absolute location (percentage correct and mean absolute deviations of judged location in relation to presented location) and of relative location (both horizontal and vertical relative positioning of each object in respect to others). These results, which do not satisfy the criteria for automaticity suggested by Hasher and Zacks $(1979,1984)$, are at odds with the claim that memory for spatial location is exclusively mediated by automatic encoding processes.

However, as Naveh-Benjamin (1987) noted, the results of his study, as well as other recent failures to discover automatic processes (e.g., frequency judgments: Greene, 1984, 1986; Naveh-Benjamin \& Jonides, 1985, 1986; Sanders, Gonzalez, Murphy, Liddle, \& Vitina, 1987) could be attributed, at least partially, to the inappropriateness of the unit of analysis. It is quite obvious that there are many processes involved in memorizing spatial location information. The recall task used in the NavehBenjamin (1987) study was quite complex in that subjects were asked to reconstruct the whole matrix of objects by placing each object in its original position. Such a task might involve several processes at retrieval, such as searching memory for location information, retrieving several items first, and then reconstructing the locations for the other items. Some of these processes might be effortful (strategic) so that even though encoding of spatial location is automatic, the use of a recall task might cover it. This might be especially true for the criteria of developmental changes and individual differences, since there is evidence that elderly subjects show deficits in tasks requiring active retrieval (Burke \& Light, 1981; Craik, 1985). Similarly, individual differences in recall of spatial location (Naveh-Benjamin, 1987) might be the results of differences between individuals in retrieval rather than in encoding processes.

To increase the likelihood that the hypothesis evaluated is about the automatic encoding of spatial location information, the following four experiments employed a simple recognition test of spatial location in which subjects had to judge which of the objects were presented in their original spatial location. Such a test, in which some of the objects presented in their original positions could reestablish the encoding context, minimizes the effects of active search on the retrieval task and probably requires less strategic processing. Five criteria for automaticity were evaluated to determine whether or not spatial location information is encoded automatically.

\section{EXPERIMENT 1}

In Experiment 1, the effects of knowledge of intention and age of subjects on recognition memory of spatial location were evaluated.

\section{Method}

Subjects. Sixty-eight subjects participated in Experiment 1. Thirty-four were students at Ben-Gurion University of the Negev, 
who participated in this experiment as part of the curriculum requirement. They ranged in age from 19 to 32 (median $=24$ ). The other 34 subjects were community-dwelling elderly people, all of whom were 65 years of age and older (median $=70$ ). According to self-reports, all of the elderly were in good physical and mental health. The majority of this group lived with their spouses. The mean number of formal years of schooling was 12.3 for the elderly group and 12.7 for the younger group.

Design. The experiment consisted of two phases. During Phase 1, subjects were presented with a $6 \times 6$ matrix of locations, 20 of which contained to-be-remembered items (see Mandler et al., 1977). One variable in the experiment was the intention to learn: half of the subjects (the incidental learning condition group) were told to rate each object in terms of its everyday usefulness. They were led to believe that this information was needed for further research and were told nothing about the subsequent memory test. The other half (the intentional learning condition group) were told about the rating task and were forewarned that a recognition memory test would be given for the objects' spatial locations. The cover story was provided to subjects in this intentional learning condition as well, so that prior knowledge about the spatial location test could be the variable to distinguish between the groups (see Eysenck, 1982).

The other variable in the experiment was subjects' age, young or old. Half of each age group was assigned randomly to each of the learning instruction conditions. These two variables resulted in four groups of 17 subjects each, since the design was completely between subjects. Phase 2 of the experiment consisted of a spatial location recognition test given to all subjects.

Materials. Twenty drawings of common objects were used as stimuli. They were pretested among a large set of objects and chosen to be easily identifiable. No more than two objects belonged to the same conceptual category.

For the learning phase, a page containing a matrix of 36 locations (each approximately $8 \mathrm{~cm}$ square) was generated in six rows and six columns. From this set of locations, several sets of 20 locations were randomly selected, with the restrictions that no more than 4 occur in any given row or column and that 5 occur within each quadrant of the matrix. The $\mathbf{2 0}$ drawings were then randomly assigned to the 20 locations, which varied from subject to subject, so that each item occupied each location in the matrix equally often in each of the four conditions of the experiment. For the recognition test phase, 10 of the objects were relocated in the following manner: 4 were moved one position, 4 were moved two positions, and 2 were moved three positions (all changes were in Euclidean distance). Half of the relocated objects for each distance were moved to previously unoccupied positions. The resulting overall distribution of occupied locations was fairly similar to the original one. The other 10 objects were left in their original positions.

Procedure. The young subjects were tested in groups of 3-5. The older subjects were tested individually to decrease anxiety and to allow more time for Phase 2 and for any further clarifications needed.

The subjects in each group were read the appropriate instructions. They were then exposed to the array for $90 \mathrm{sec}$, during which time they were to look at each object and rate its usefulness by writing a number from 1 (not useful at all) to 5 (very useful) near it. Starting with any object at will, they were asked to rate all objects, without exception, which all did. In the intentional group they were told to study the spatial location of each object while completing the rating task. The time allowed was sufficient for all subjects. Following this phase, the experimenter engaged the subjects in approximately $3 \mathrm{~min}$ of conversation before Phase 2 of the experiment began.

The subjects were then given the instructions and materials for the test phase. In this phase they were given the test matrix and were told that the location of 10 of the objects was changed, and that their task was to mark the 10 objects whose location was the same as in the original matrix. To assure an unbiased criterion measure, the subjects were specifically instructed to mark exactly 10 objects, which they did. No time limit was set for this phase, although the subjects completed the task in a short time. After completion, the subjects were given a questionnaire about their understanding of the experiment, the extent to which they followed the instructions, their expectations about the spatial location test, and their motivation to perform well in the experiment.

\section{Results and Discussion}

There were no differences between the groups with respect to understanding the instructions and motivation to succeed. For each subject, the percentage of objects correctly recognized in their original spatial locations was calculated. The resulting group means are presented in Table 1. Those subjects in the intentional learning groups performed better, and the younger subjects performed better. ${ }^{1}$ These differences were confirmed in a two-way analysis of variance (ANOVA) that showed the effects of learning instructions and age to be significant $[F(1,64)$ $=18.22$ and $F(1,64)=36.23$, respectively]. (An alpha level of $p<.05$ is used for all tests reported in this article.) However, the effect of the interaction of the two variables was not significant $[F(1,64)=0.001, M S e=$ 155.5]. The results are clear: Memory for spatial location information is influenced by the intention of the subjects, and it declines in old age. ${ }^{2}$

It should be noted that the effect of learning instructions was probably due to differences in encoding the information since this manipulation was done in the encoding (learning) stage. The results for age showed the same age deficits as those reported by Naveh-Benjamin (1987) for a recall test even when a recognition test requiring less active retrieval was used. This, in turn, could indicate the influence of encoding processes on the age differences obtained.

These results fail to confirm two major criteria offered for the identification of automatic processes; this leads one to question the suggestion that encoding of spatial location might be mediated by an automatic process.

\section{EXPERIMENT 2}

The purpose of Experiment 2 was to test a third criterion suggested for the identification of automatic processes, the claim that such processes are not disrupted by simultaneous processing demands. To test this criterion in an optimal way, such a test should include a primary task that requires attention to spatial location of the various objects and a secondary task that varies in

Table 1

Percentage of Correct Recognition as a Function of Age of Subjects and Intention to Learn

\begin{tabular}{lccccc}
\hline & \multicolumn{2}{c}{ Young } & & \multicolumn{2}{c}{ Old } \\
\cline { 2 - 3 } \cline { 5 - 6 } & $M$ & $S D$ & & $M$ & $S D$ \\
\hline Incidental & 69.4 & 10.3 & & 51.2 & 15.3 \\
Intentional & 82.3 & 12.0 & & 64.1 & 11.2 \\
\hline
\end{tabular}


difficulty. Effects of secondary task load on performance of the primary task would establish an effect of competing demands. Furthermore, by varying secondary task difficulty and finding an effect of this variation, one could be confident that the interference of the secondary task on the primary task was caused by more than "structural interference" (see Kahneman, 1973; Posner \& Boies, 1971). Thus, an experiment of this sort would be relevant to the issue of whether spatial location coding meets the criterion of insensitivity to competing load. (See Naveh-Benjamin \& Jonides, 1986, for a similar argument in the context of coding of frequency of occurrence.)

The following experiment met this need. The subjects were required to engage in a digit-counting task while preparing for a recognition test of spatial location of objects. The load of the secondary task was varied by changing the difficulty of the counting task. In the light-load condition, the subjects had to count backward by $1 \mathrm{~s}$; in the heavy-load condition, they were asked to count backward by multiples of 13 .

\section{Method}

Subjects. The subjects were 48 undergraduate students at the BenGurion University of the Negev, who participated as part of their curriculum requirement.

Materials. The materials were identical to those of Experiment 1.

Procedure. Twenty-four subjects were assigned randomly to each of the experimental conditions and tested in groups of 4-10. Before the study phase of the experiment, the subjects were told that they would have to perform two tasks while being exposed to the matrices with the objects. The first task was to pay attention to the pictures of the objects in preparation for the upcoming spatial location recognition memory test. The second task was to write down a number near each object surveyed in the matrix, starting with a specific number. The subjects in one group were told to count backward by $1 \mathrm{~s}$, starting with the number 1,423 . The subjects in the second group were told to count backward by $13 \mathrm{~s}$, starting with 1,548. (The starting numbers were chosen to yield the same average number-1,413-for both conditions.) The subjects in both groups were asked to pay equal attention to the spatial location of the objects and to the counting task at hand. After some practice in counting, the subjects made practically no errors in the counting task. The difficulty of the counting task was tested in a preliminary study, in which 16 subjects were asked to count backward as fast as they possibly could by $1 \mathrm{~s}$ or by $13 \mathrm{~s}$ (order of the tasks was counterbalanced) for $1 \mathrm{~min}$. The mean number of digits counted was 24.6 and 13.1 for the respective tasks. These differences turned out to be significant $[t(15)=6.2]$. The results assured us that the difficulty of the counting tasks was as hypothesized. During the study phase of the experiment, the subjects studied the objects of the matrix while counting backward for $2 \mathrm{~min}$. During pretesting, we established this duration as sufficiently long for completing the task in both conditions. All subjects indicated that they had looked at all the objects presented and all finished writing one number near each object reviewed, although the order in which the matrix was surveyed varied across subjects. Following this phase and a 3 -min conversation period, the recognition test for spatial location was administered as in Experiment 1.

\section{Results and Discussion}

As in Experiment 1 for each subject, the percentage of objects recognized correctly in their original positions was calculated. The resulting means across subjects in each group are presented in Table 2 . The heavy task load resulted in poorer performance. This trend was confirmed by a $t$ test that showed the differences between conditions to be significant $[t(46)=2.39]$. The results are clear: the heavier the load of the competing task is, the worse the recognition memory of spatial location. These results are not consistent with the claim that spatial location coding is automatic.

It should be noted that the effect of load was not due to a decrease in the time left for processing of spatial location information after the simultaneous counting task was completed, as indicated by pilot studies in which presentation time was manipulated from 60 to $120 \mathrm{sec}$ under intentional instructions with minor observable effects on performance. Instead, the effects of load could be more directly attributed to either momentary decreased capacity devoted to spatial coding or to less effective strategies used with heavier competing demands.

\section{EXPERIMENT 3}

In Experiment 3 we focused on a fourth criterion of automatic processes, the lack of practice effect. To test the influence of practice on the encoding of spatial location information, a group of subjects was involved in two identical consecutive tasks requiring memorization of spatial location information, and an evaluation was made as to whether there were any practice effects. In the first task, the subjects run under intentional learning instructions performed without any previous experience in doing a similar task. After having the experience of encoding and memorizing spatial location information in such a task, they participated in a second, similar task. If the hypothesis about the automaticity of encoding spatial location information has some merit, there should be no performance improvement in the second task due to practice gained in the first task. This manipulation of practice is quite weak, since the observation of the effects on performance takes place after a very brief practice regime. Nevertheless, the manipulation satisfactorily showed significant effects on performance.

\section{Method}

Subjects. Subjects were 21 students at Ben-Gurion University of the Negev who participated as part of their curriculum requirement.

Materials. Two sets of objects were prepared for use in the matrix. One set included the objects used in the previous experiments. The other was constructed according to the same guidelines used for the first set; the objects chosen were equated to those used in the first set in terms of their frequency in language, imaginability, and semantic categorical affiliation.

Procedure. The subjects were run under intentional learning conditions for both tasks. Half the subjects were randomly assigned to use the first set of objects in the first task, and the other half used the second set of objects in the first task. The subjects were told to study the spatial location of the objects to prepare for an upcoming spatial location recognition test for the objects. After finishing the study and test of the first matrix, they were engaged in 5-min conversation, after which they performed the same task 
with the second set of objects, using the same procedures as those employed in the first task. The subjects were allowed $90 \mathrm{sec}$ for studying the matrix in each phase of the experiment.

\section{Results and Discussion}

For each subject, the percentage of objects recognized correctly in their original spatial locations was calculated. The mean values for this measure for each trial are shown in Table 2. Performance on the second trial following training was better than performance on the first trial prior to training. These differences were shown to be statistically significant $[t(20)=2.26]$.

These results imply that, despite the very short and superficial practice in memorizing spatial location information, the subjects improved their performance in a task that requires the encoding of spatial location information. These results contradict claims about the automatic nature of spatial location coding.

\section{EXPERIMENT 4}

Experiment 4 focused on a fifth criterion of automatic processes, the lack of individual differences. In this experiment, individual differences were examined in a way similar to that used by Zacks, Hasher, and Sanft (1982) in the context of frequency judgments, by comparing performance of students from two different departments at Ben-Gurion University of the Negev. Department A uses highly selective admission standards, whereas Department $B$ uses more liberal standards. The difference between the median scores of students in the two departments on University National Entrance Examinations exceeds two standard deviations. If spatial location information is automatically encoded, it is expected that any differences in motivation to perform well, systematic use of mnemonic strategies, and intelligence that these scores may reflect should not influence recognition memory for the spatial location task.

\section{Method}

Subjects. The subjects were 62 students at Ben-Gurion University of the Negev, 38 of whom studied in Department A and 24 in Department B. Both groups of students participated as part of a curriculum requirement. None of these students participated in any of the previous experiments.

Materials. The materials were identical to the ones used in Experiment 1.

Procedure. The subjects were tested under intentional instructions in which they were told to study the spatial position of each of the presented objects to prepare for a recognition test on these locations. After studying the matrix with the objects for $90 \mathrm{sec}$, they were engaged in a 3 -min conversation, after which they were given the recognition test for spatial location, the same as in previous experiments. They were allowed as much time as they needed for completing the test. The subjects were tested in groups of 4-7.

\section{Results and Discussion}

For each subject, the percentage of objects recognized correctly in their original spatial locations was calculated. The mean values for this measure for students of each
Table 2

Percentage of Correct Recognition as a Function of Load

Condition (Experiment 2), Practice (Experiment 3), and Students' Departmental Affiliation (Experiment 4)

\begin{tabular}{lccc}
\hline & $M$ & $S D$ & $N$ \\
\hline Light Load (-1) & 77.0 & 13.0 & 24 \\
Heavy Load (-13) & 67.5 & 13.9 & 24 \\
Practice, Trial 1 & 78.6 & 10.1 & 21 \\
Practice, Trial 2 & 83.8 & 12.0 & 21 \\
Department A & 81.6 & 10.5 & 38 \\
Department B & 75.5 & 11.3 & 24 \\
\hline
\end{tabular}

department are shown in Table 2. The results are clear: Students in Department A performed better than those in Department B. These differences were significant $[t(60)$ $=2.13]$.

These results show that students studying in the more selective department performed better on a task involving recognition memory for spatial location information. Such results imply that differences among individuals have marked effects on memory for spatial location information. The results fail to confirm one of the criteria suggested for testing automaticity of cognitive processes. The current results, which show the same individual differences in recognition memory as those reported by NavehBenjamin (1987) for recall memory, decrease the likelihood that the differences obtained are due to active processes at retrieval.

\section{GENERAL DISCUSSION}

The results of all four experiments are clear. Experiment 1 shows that when intentionality is appropriately defined and operationalized, the encoding of spatial location information is influenced by the intention to learn. Subjects who learn the information intentionally more accurately recognize the spatial locations of the objects than subjects who incidentally learn the information. In addition, the encoding of spatial location information is a function of subjects' ages. Older subjects do not recognize spatial location as well as younger subjects. These results are at odds with two major criteria suggested by Hasher and Zacks $(1979,1984)$ for testing whether a given task is based on the operation of automatic processes. Note, however, that the interaction of these two variables did not reach significance, implying a possible lack of qualitative differences between young and old subjects in the strategies employed under the various intention conditions.

The results of Experiment 2 show that the encoding of spatial location information is influenced by competing task demands. Increasing the load of a competing task causes a decline in the accuracy of recognition memory for objects' spatial locations. These results are also at odds with one of the main criteria suggested for testing the automaticity of cognitive operations.

The results of Experiment 3 show that the encoding of spatial location information is influenced by practice. Applying a short training regime results in a significant improvement in recognition of spatial location information. 
The results of Experiment 4 show that the encoding of spatial location information is influenced by individual differences. Students studying in a department with more selective admissions standards were more accurate in recognizing spatial location information. These results are also at odds with one of the criteria suggested for testing for the automaticity of cognitive processes.

Altogether, the picture emerging from this work is clear. The results show that none of the criteria tested for memory for spatial location information was fulfilled, in contrast to the claim that spatial location information is automatically encoded. ${ }^{3}$ This data was obtained even when a recognition memory task, which minimizes the effects of active retrieval and reflects mostly encoding differences, was used.

These results support the ones obtained by NavehBenjamin (1987) with recall tasks. The results of the current study agree with recent results reported for another type of information suggested as being automatically coded, namely, frequency information. Jonides and Naveh-Benjamin (1987), Naveh-Benjamin and Jonides (1985, 1986), Maki and Ostby (1987), and Sanders, Gonzalez, Murphy, Liddle, and Vitina (1987) have shown that frequency coding is influenced by competing task load and strategy manipulation. In addition, Fisk and Schneider (1984), Greene (1984), Jonides and Naveh-Benjamin (1987), Naveh-Benjamin and Jonides $(1985,1986)$, and Sanders et al. (1987) have shown that the encoding of frequency information is sensitive to intentional manipulations.

The question then arises: Is the coding of spatial location information automatic? There are several avenues for answering this question. First, the results obtained in the current experiments and in the recent ones on frequency coding raise some doubts about automatic and effortful processes being distinct, as once was believed. It could be that there is a continuum linking automatic and nonautomatic processes (see Zbrodoff \& Logan, 1986, who recently suggested that various properties of automaticity, e.g., effort and autonomy, could be conceived as continuous dimensions). The difference among points on this continuum may be only a matter of degree. If such is the case, then our results imply that spatial location coding is certainly not at the extremely automatic end of this continuum, as previously argued (and it is neither autonomous nor effortless according to Zbrodoff \& Logan, 1986). Where it is located cannot be addressed without a principled understanding of how the criteria suggested for automatic processes can be used to order the processes on the continuum according to the number of criteria satisfied. Moreover, recent results about the major processes suggested by Hasher and Zacks $(1979,1984)$ as automatically operating (Fisk \& Schneider, 1984; Greene, 1984; Naveh-Benjamin \& Jonides, 1985, 1986; Sanders et al., 1987, for frequency information; Zacks, Hasher, Alba, Sanft, \& Rose, 1984, for temporal order information), and those of the current study for spatial location infor- mation, show that there is currently no process that could be placed near the automatic end of the continuum.

A second answer to the question of automaticity of spatial location coding concerns the adequacy of the criteria suggested for automatic processes. Naveh-Benjamin (1987) recently evaluated the criteria for automaticity suggested by Hasher and Zacks $(1979,1984)$ and showed them to be too strong. He has shown serious problems with the assumption underlying these criteria, which claims continuous optimal function of automatic processes under all circumstances (Hasher \& Zacks, 1984). This association of automaticity with optimal performance cannot easily be defended (see Naveh-Benjamin, 1987, for a detailed discussion).

What criteria could then be used to distinguish automatic from nonautomatic processes? Sanders et al. (1987) recently suggested more lenient criteria for automaticity in the context of tests of memory for frequency of occurrence. Unable to support some of the criteria suggested by Hasher and Zacks, these researchers proposed a modified definition of automatic processes. According to this definition, automatic processing should be capable of producing an above-chance output while requiring no more than minimal resources and little or no conscious awareness. Although this definition is clearly more relaxed than the one put forward by Hasher and Zacks, it is also more difficult to operationalize. The major objective empirical test for automaticity of a process, according to this definition, is an above-chance performance.

This criterion, however, is not completely new; it is a variant of the first criterion suggested by Hasher and Zacks, namely that "people are sensitive to the information claimed to be automatically encoded without necessarily intending to be so" $(1984$, p. 1373). In addition, Park and Mason (1982) already suggested the use of such criterion in the context of tests of automaticity of spatial location information. However, as already noted by Naveh-Benjamin (1987), this criterion is problematic since there is ample evidence that the performance of subjects in tasks considered to involve effortful processes, such as free recall, is at a better-than-chance level even under true incidental learning instructions (e.g., Hyde \& Jenkins, 1969).

From this perspective, most cognitive processes could be considered automatic. On the other hand, even the two widely used examples of automatic processes, those involved in coding frequency and spatial location information, might not be considered automatic by this criterion. Naveh-Benjamin and Jonides (1985) have shown that under conditions involving minimal resources directed toward processing frequency information (in a maintenance rehearsal paradigm), frequency judgments are not above chance. In addition, Naveh-Benjamin (1987) has shown that under heavy concurrent task load, recall of spatial location memory is not above chance. Finally, in Experiment 1 of this report, performance of elderly subjects under incidental learning instructions was not above 
chance. To summarize, above-chance performance as an alternative criterion for automatic processing also might be problematic.

A possible direction, at least for fairly complex tasks such as memorization of spatial location, will be to hold to those criteria suggested by Hasher and Zacks, but in a weaker sense. In this sense, the processes showing less noticeable changes as a function of age, practice, simultaneous processing, and so forth, could be considered as more automatic than the others. This direction naturally coincides with the continuum idea previously discussed. Although this concept might be theoretically interesting, there still remains to be seen whether there is a way of operationalizing placements of various tasks on such a continuum, which will involve devising methods of allowing comparisons of performance across different task scales.

\section{REFERENCES}

ACredolo, L. P., Pick, H. L., JR., \& Olsen, M. G. (1975). Environmental differentiation and familiarity as determinants of children's memory for spatial location. Developmental Psychology, 11, 495-501.

Burke, D. M., \& Light, L. L. (1981). Memory and aging: The role of retrieval processes. Psychological Bulletin, 90, 513-546.

Craik, F. I. M. (1985). Paradigms in human memory research. In L.-G. Nilsson \& T. Archer (Eds.), Perspectives on learning and memory (pp. 197-221). Hillsdale, NJ: Erlbaum.

EysenCK, M. W. (1982). Incidental learning and orienting tasks. In C. R. Puff (Ed.), Handbook of research methods in human memory and cognition (pp. 197-228). New York: Academic Press.

FisK, A. D., \&CHNeIDER, W. (1984). Memory as a function of attention, level of processing, and automatization. Joumal of Experimental Psychology: Learning, Memory, \& Cognition, 10, 181-197.

GrEenE, R. L. (1984). Incidental learning of event frequency. Memory \& Cognition, 12, 90-95.

GrRENE, R. L. (1986). Effects of intentionality and strategy on memory for frequency. Journal of Experimental Psychology: Learning, Memory, \& Cognition, 12, 489-495.

Hasher, L., ZACKs, R. T. (1979). Automatic and effortful processes in memory. Journal of Experimental Psychology: General, 108, 356-388.

HASHER, L., \& ZACKs, R. T. (1984). Automatic processing of fundamental information: The case of frequency of occurrence. American Psychologist, 39, 1372-1388.

Herman, J. F., Kolker, R. G., Shaw, M. L. (1982). Effects of motoractivity on children's intentional and incidental memory for spatial locations. Child Development, 53, 239-244.

HydE, T. S., JENxINs, J. J. (1969). Differential effects of incidental tasks on the organization of recall of a list of highly associated words. Joumal of Experimental Psychology, 82, 472-481.

JoNIDES, J. (1981). Voluntary versus automatic control over the mind's eye's movement. In J. Long \& A. D. Baddeley (Eds.), Attention and performance $I X$ (pp. 187-204). Hillsdale, NJ: Erlbaum.

JONIDES, J., NAVEH-BENJAMIN, M. (1987). Estimating frequency of occurrence. Joumal of Experimental Psychology: Learning, Memory, \& Cognition, 13, 230-240.

Kahneman, D. (1973). Attention and effor. Englewood Cliffs, NJ: Prentice-Hall.

LIGHT, L. L., ZeLINSKI, E. M. (1983). Memory for spatial information in young and old adults. Developmental Psychology, 19, 901-906.

Lovelace, E. A., \& Southall, S. D. (1983). Memory for words in prose and their location on the page. Memory \& Cognition, 11, $429-434$.
MAKI, R. H., \& OSTBY, R. S. (1987). Effects of level of processing and rehearsal on frequency judgments. Journal of Experimental Psychology: Learning, Memory, \& Cognition, 13, 151-163.

Mandler, J. M., Seegmiller, D., DAy, J. (1977). On the coding of spatial information. Memory \& Cognition, 5, 10-16.

McCormack, P. D. (1982). Coding of spatial information by young and elderly adults. Joumal of Gerontology, 37, 80-86.

NAveh-Benjamin, M. (1987). Coding of spatial location information: An automatic process? Joumal of Experimental Psychology: Learning, Memory, \& Cognition, 13, 595-605.

NAVEh, Benjamin, M., Jonides, J. (1984). Maintenance rehearsal A two-component analysis. Journal of Experimental Psychology: Learning, Memory, \& Cognition, 10, 369-385.

Naveh-Benjamin, M., Jonides, J. (1985). The effects of rehearsal on frequency coding. Bulletin of the Psychonomic Society, 23, 387-390.

Naveh-Benjamin, M., \& Jonides, J. (1986). On the automaticity of frequency coding: Effects of competing task load, encoding strategy, and intention. Journal of Experimental Psychology: Leaming, Memory, \& Cognition, 12, 378-386.

PARK, D. C., JAMES, C. Q. (1983). Effect of encoding instructions on children's spatial and color memory: Is there evidence for automaticity? Child Development, 54, 61-68.

PARK, D. C., \& MASON, D. A. (1982). Is there evidence for automatic processing of spatial and color attributes present in pictures and words? Memory \& Cognition, 10, 76-81.

Parks, D. C., Puglisi, J. T., \& Lutz, R. (1982). Spatial memory in older adults: Effects of intentionality. Journal of Gerontology, 37, 330-335.

Perlmutter, M., Metzger, R., Nezworski, T., \& Miller, K. (1981). Spatial and temporal memory in 20 and 60 year olds. Joumal of Gerontology, 36, 59-65.

Pezdek, K. (1983). Memory for items and their spatial locations by young and elderly adults. Developmental Psychology, 19, 895-900.

Pezdek, K., Roman, Z., Sobolik, K. G. (1986). Spatial memory for objects and words. Journal of Experimental Psychology: Learning, Memory, \& Cognition, 12, 530-537.

Posner, M. I., \& Boies, S. J. (1971). Components of attention. Psychological Review, 78, 391-408.

Posner, M. I., \& SNyder, C. R. (1975). Attention and cognitive control. In R. Solso (Ed.), Information processing and cognition: The Loyola Symposium (pp. 55-86). Hillsdale, NJ: Erlbaum.

Sanders, R. E., Gonzalez, E. G., MurPhy, M. D., Liddle, C. L., \& VitinA, J. R. (1987). Frequency of occurrence and the criteria for automatic processing. Journal of Experimental Psychology: Learning, Memory, \& Cognition, 13, 241-250.

Schneider, W., Shiffrin, R. N. (1977). Controlled and automatic human information processing: I. Detection, search, and attention. Psychological Review, 84, 1-66.

Schulman, A. I. (1973). Recognition memory and the recall of spatial location. Memory \& Cognition, 1, 256-260.

ShIFFrIn, R. M., SCHNeIDER, W. (1977). Controlled and automatic human information processing: II. Perceptual learning, automatic attending, and a general theory. Psychological Review, 84, 127-190.

von Wright, J. M., Gebhard, P., \& KarTtunen, M. (1975). A developmental study of the recall of spatial location. Journal of Experimental Child Psychology, 20, 181-190.

WADDELL, K. J., RoGOFF, B. (1981). Effect of contextual organization on spatial memory of middle-aged and older women. Developmental Psychology, 17, 878-885.

Zacks, R. T., Hasher, L., Alba, J. W., Sanft, H., \& Rose, K. C. (1984). Is temporal order encoded automatically? Memory \& Cognition, 12, 387-394.

Zacks, R. T., Hasher, L., SAnft, H. (1982). Automatic encoding of event frequency: Further findings. Joumal of Experimental Psychology: Learning, Memory, \& Cognition, 8, 106-116.

ZBrodoFF, N. J., LoGAN, G. D. (1986). On the autonomy of mental processes: A case study of arithmetic. Joumal of Experimental Psychology: General, 115, 118-130. 
Zechmeister, E. B., McKillip, J., Pasko, S., Bespalec, D. (1975) Visual memory for place on the page. Journal of General Psychol ogy, 92, 43-52.

\section{NOTES}

1. It should be noted that although the elderly subjects in the incidental condition performed at chance level, it probably did not reflect lack of retention of any information from the experiment. In a pilot study, a group of elderly possessing the same characteristics showed appreciable item memory in a similar task. In addition, the advantage of the young over the old subjects was probably not due to the fact that the younger subjects were nun in groups, which might have facilitated performance: in pilot research, there were no differences in performance between young subjects run in small groups and those run individually.
2. The error data in all experiments showed the same pattern of inverse relations with the degree of relocation of objects in the recognition test, so that subjects committed fewer errors with objects that were relocated a farther distance. This pattern did not interact with the manipulations of any of the experiments.

3. It should be noted that these results were obtained while using pictures of objects for which we expect the automaticity claim to hold, in contrast to the case in which verbal labels of objects are used (see Pezdek, Roman, \& Sobolik, 1986).

(Manuscript received September 24, 1987; revision accepted for publication January 5,1988 .) 